\title{
Selective Interaction of Sugarcane eIF4E with VPgs from Sugarcane Mosaic Pathogens
}

\author{
Zongtao Yang 1,2, Meng Dong 1,2, Guangyuan Cheng 1,2, Shuxian Liu 1,2, Hai Zhang 1,2, Heyang Shang 1,2, \\ Yingshuan Zhou ${ }^{1,2}$, Guoqiang Huang ${ }^{1,2}$, Muqing Zhang ${ }^{3}$, Fengji Wang ${ }^{2, *}$ and Jingsheng $X u^{1,2, *}$ (i) \\ 1 National Engineering Research Center for Sugarcane \& Key Laboratory of Sugarcane Biology and Genetic Breeding, \\ Ministry of Agriculture and Rural Affairs, Fujian Agriculture and Forestry University, Fuzhou 350002, China; \\ YZT@fafu.edu.cn (Z.Y.); dongm6174@163.com (M.D.); chengguangyuanfafu@163.com (G.C.); \\ liushuxian1010@163.com (S.L.); zhanghai940410@163.com (H.Z.); heyangshang@hotmail.com (H.S.); \\ zhouyingshuan123@126.com (Y.Z.); hgq94@163.com (G.H.) \\ 2 Key Laboratory of Ministry of Education for Genetics, Breeding and Multiple Utilization of Crops, \\ College of Crop Science, Fujian Agriculture and Forestry University, Jinshan, Fuzhou 350002, China \\ 3 State Key Laboratory for Conservation and Utilization of Agro-Bioresources, Guangxi Key Laboratory for \\ Sugarcane Biology, Guangxi University, Nanning 530005, China; zmuqing@163.com \\ * Correspondence: wangfengji@fafu.edu.cn (F.W.); xujingsheng@fafu.edu.cn (J.X.)
}

Citation: Yang, Z.; Dong, M.; Cheng, G.; Liu, S.; Zhang, H.; Shang, H.; Zhou, Y.; Huang, G.; Zhang, M.; Wang, F.; et al. Selective Interaction of Sugarcane eIF4E with VPgs from Sugarcane Mosaic Pathogens. Viruses 2021, 13, 518. https://doi.org/ $10.3390 / \mathrm{v} 13030518$

Academic Editor: K. Andrew White

Received: 6 March 2021

Accepted: 20 March 2021

Published: 22 March 2021

Publisher's Note: MDPI stays neutral with regard to jurisdictional claims in published maps and institutional affiliations.

Copyright: (c) 2021 by the authors. Licensee MDPI, Basel, Switzerland. This article is an open access article distributed under the terms and conditions of the Creative Commons Attribution (CC BY) license (https:// creativecommons.org/licenses/by/ $4.0 /)$.

\begin{abstract}
Eukaryotic translation initiation factor 4E (eIF4E) plays a key role in the infection of potyviruses in susceptible plants by interacting with viral genome-linked protein (VPg). Sugarcane (Saccharum spp.) production is threatened by mosaic disease caused by Sugarcane mosaic virus (SCMV), Sorghum mosaic virus (SrMV), and Sugarcane streak mosaic virus (SCSMV). In this study, two eIF4Es and their isoform $e I F($ iso $) 4 E$ and $4 \mathrm{E}$-binding protein coding genes were cloned from sugarcane cultivar ROC22 and designated SceIF4Ea, SceIF4Eb, SceIF(iso) $4 E$, and ScnCBP, respectively. Real-time quantitative PCR analysis showed different expression profiles of these four genes upon SCMV challenge. A subcellular localization assay showed that SceIF4Ea, SceIF4Eb, SceIF(iso)4E, and ScnCBP were distributed in the nucleus and cytoplasm. Yeast two-hybrid ( $\mathrm{Y} 2 \mathrm{H})$ and bimolecular fluorescence complementation (BiFC) assays showed that SceIF4Ea/b and SceIF(iso)4E were selectively employed by different sugarcane mosaic pathogens, i.e., SCMV-VPg interacted with SceIF4Ea/b and SceIF(iso)4E, SrMV-VPg interacted with both SceIF4Eb and SceIF(iso)4E, and SCSMV-VPg interacted only with SceIF(iso)4E. Intriguingly, the BiFC assays, but not the $\mathrm{Y} 2 \mathrm{H}$ assays, showed that ScnCBP interacted with the VPgs of SCMV, SrMV, and SCSMV. Competitive interaction assays showed that SCMV-VPg, SrMV-VPg, and SCMV-VPg did not compete with each other to interact with SceIF(iso)4E, and SceIF(iso)4E competed with SceIF4Eb to interact with SrMV-VPg but not SCMV-VPg. This study sheds light on the molecular mechanism of sugarcane mosaic pathogen infection of sugarcane plants and benefits sugarcane breeding against the sugarcane mosaic disease.
\end{abstract}

Keywords: eIF4E; VPg; SCMV; SrMV; SCSMV

\section{Introduction}

Sugarcane (Saccharum sp.) is the most important sugar and energy crop worldwide [1-3]. Sugarcane mosaic disease is widespread in sugarcane-growing countries [4-15] and causes heavy yield losses [16,17]. In the 1920s, sugarcane mosaic disease almost collapsed the sugar industry in Argentina, Brazil, and Louisiana [18]. Although sugarcane mosaic disease is controlled by planting resistant sugarcane cultivars, it continues to be a threat to the sugarcane industry $[4,15,17,19]$. The main causal agents for the sugarcane mosaic disease are Sugarcane mosaic virus (SCMV), Sorghum mosaic virus (SrMV), and Sugarcane streak mosaic virus (SCSMV), which belong to the Potyviridae family, with SCMV and SrMV being members of the Potyvirus genus [20], whereas SCSMV is a member of Poacevirus genus [21-23]. Although SCMV, SrMV, and SCSMV belong to different genera in Potyviridae, they share very similar 
genomic structures. They all have a single-stranded positive-sense $10 \mathrm{~kb}$ RNA genome encoding 2 polyproteins that are then proteolytically processed into 11 mature proteins: P1, helper component proteinase (HC-Pro), P3, P3N-PIPO, 6K1, cylindrical inclusion (CI), 6K2, viral genome-linked protein (VPg), proteinase domain of NIa (NIa-Pro), nuclear inclusion protein b $(\mathrm{NIb})$, and $\mathrm{CP}$ [24-32].

In eukaryotic organisms, mRNA translation is predominantly cap dependent, and recognition of the cap structure $\left(\mathrm{m}^{7} \mathrm{GpppN}\right.$, where $\mathrm{N}$ is any nucleotide) at the $5^{\prime}$ terminus of mRNA is a key step in the cellular regulation of translation [33,34]. Translation initiation is a multistep process involving the assembly of a mRNA-protein complex by different eukaryotic translation initiation factors (eIFs). The binding of eIF4E to the cap structure of a mRNA is key for translation. Gaining access to the host translation machinery is essential for viruses in the Potyviridae family to establish infection in the host $[25,35,36]$. However, for the positive-sense RNA plant viruses in the Potyviridae and Secoviridae families and some members in the Luteoviridae family and Sobemovirus genus, there is no cap structure at the $5^{\prime}$ end of the genome RNA $[25,37]$. VPg serves as an analog of the cap structure by covalently linking to the $5^{\prime}$ end of virus sense genome RNA via a tyrosine or serine residue [38-41]. It has been well documented that the VPg of potyviruses interacts with the host cap-binding eIF4E or eIF(iso)4E proteins to initiate viral genome translation $[25,35,37,42,43]$.

Silencing of eIF4E, eIF(iso) $4 E$ or $n C B P$ contributes to mutant plant resistance to one or more species of viruses. In Arabidopsis thaliana, eIF(iso) $4 E$ mutant plants are resistant to Turnip mosaic virus (TuMV), Tobacco etch virus (TEV) or Lettuce mosaic virus (LMV) [44,45]. The eIF4E-mutant plants are resistant to Clover yellow vein virus (CIYVV) [46]. In pepper, the knockout mutation of eIF4E causes resistance to Potato virus $Y$ (PVY) and TEV [47,48]. The mutant of lettuce eIF4E is resistant to Lettuce mosaic virus (LMV) [49]. In tomato, knockdown of eIF4E1 confers resistance to both PVY and Pepper mottle virus (PepMoV) [50]. Further, knockdown of both the eIF4E1 and eIF4E2 genes confers broad-spectrum resistance against PVY, TEV, Pepper mottle virus (PepMoV), Ecuadorian rocotto virus (ERV), Pepper severe mosaic virus (PepSMV), Pepper yellow mosaic virus (PepYMV), and Potato virus V (PVV) in mutant tomato plants [51]. Furthermore, most of the mutant lines show no growth defects. A study of these mutants led to the discovery of the molecular nature of plant recessive resistance to plant viruses [42,52-54]. To date, 14 natural recessive resistance genes corresponding to eIF4E or its isoform $e I F($ iso $4 E$ against plant viruses have been identified from diverse plant species $[37,55,56]$.

The molecular mechanism of sugarcane mosaic pathogen infection in sugarcane is poorly understood [15,17,30,57-59] due to the highly complex sugarcane genome and difficulty of performing transformation $[59,60]$. In the present study, we cloned 4 eIF4E homologs obtained from sugarcane cultivar ROC22 and designated them as SceIF4Ea, SceIF4Eb, SceIF(iso) $4 E$, and ScnCBP. Their expression profiles upon the SCMV challenge were investigated by real-time quantitative PCR, and the interaction with SCMV-/SrMV/SCSMV-VPg was individually explored by yeast two-hybrid (Y2H) and bimolecular fluorescence complementation (BiFC) assays. This study is helpful for understanding the molecular mechanism of SCMV, SrMV or SCSMV infection of sugarcane and instructive for the molecular breeding for sugarcane resistance to mosaic diseases.

\section{Materials and Methods}

\subsection{Plant Materials and Viruses}

SCMV, SrMV, and SCSMV isolates were provided by the Key Laboratory of Sugarcane Biology and Genetic Breeding, Ministry of Agriculture and Rural Affairs, P.R. China. Healthy seed canes were propagated from the axillary bud of $\mathrm{cv}$. ROC22 and exposed to a $14 / 10-\mathrm{h}$ light/dark cycle at $28^{\circ} \mathrm{C}$ in the greenhouse. ROC22 plantlets were individually inoculated with SCMV at the 5-6 leaf stage, as previously described [59], and mockinoculated ROC22 plantlets with $0.01 \mathrm{M}$ phosphate buffer $(\mathrm{pH} 7.0)$ were used as the control. The inoculated or mock inoculated leaves were sampled on $0,1,2$, and 5 days post-inoculation. All plant materials were collected in triplicate, immediately frozen in 
liquid nitrogen, and then placed in a $-80{ }^{\circ} \mathrm{C}$ refrigerator for RNA isolation. Nicotiana benthamiana plants were grown at $22 \pm 0.5{ }^{\circ} \mathrm{C}$ and $70 \%$ relative humidity under a $16-\mathrm{h}$ day/8-h night photoperiod in climate-controlled cabinets. Illumination of $90 \mu \mathrm{mol} / \mathrm{s} / \mathrm{m}^{2}$ was generated by a fluorescent lamp.

\subsection{RNA Extraction and Real-Time Quantitative PCR}

Leaf samples were ground into powder in liquid nitrogen. One hundred milligrams of leaf powder was mixed with $1 \mathrm{~mL}$ of TriPure reagent (Roche, Basel, Switzerland). Total RNA was extracted according to the manufacturer's instructions. The RNA concentration and quality were determined using an ND-1000 spectrophotometer (NanoVue Plus, GE, Chicago, IN, USA) and electrophoresis. First-strand cDNA was synthesized using a PrimeScript RT-PCR kit (TaKaRa, Dalian, China) according to the manufacturer's instructions.

Real-time quantitative PCR (RT-qPCR) was carried out using the Fast Universal SYBR Green Master mix (ROX; Roche, Hercules, CA, USA) on an ABI7500 real-time PCR system (Applied Biosystems, Foster City, CA, USA). GAPDH [61] and $e E F-1 \alpha$ were used as internal references [62], and three replicates were completed for each sample. A melting curve analysis was conducted to confirm the PCR specificity for each primer pair. The data were analyzed using the $2^{-\Delta \Delta C t}$ method to determine the altered gene expression, and the results are shown as the mean of three biological replicates with the corresponding standard error.

\subsection{Plasmid Construction}

All primers used for plasmid construction are listed in Supplemental Table S1. For the $\mathrm{Y} 2 \mathrm{H}$ experiments, the yeast two-hybrid vectors and all DNA fragments were individually ligated via SfiI sites. $S C M V-V P g, S r M V-V P g, S C S M V-V P g$, and $S c n C B P$ were individually cloned into the bait vector pGBKT7. SceIF4Ea, SceIF4Eb, SceIF(iso)4E, and ScnCBP were individually cloned into the acquired vector pGADT7. For the pBridge vector construction, $S C M V$-/SrMV-/SCSMV-VPg was individually inserted into multiple clone site 1 (MCS1) using restrictive endonuclease XmaI, while $S C M V$ - $/ S r M V-/ S C S M V$ - $V P g$, SceIF4Eb, and SceIF(iso)4E were individually inserted into MCS2 using restrictive endonuclease NotI.

For the transient protein expression and BiFC assays, all plasmids were generated using the gateway technology, as described by Cheng et al. [31]. The resulting respective DNA fragments were purified and cloned into a pDONR221 entry vector using the BP reaction according to the manufacturer's instructions. The pDONR221 vector containing the target gene was recombined into the destination vector pEarleyGate201-YN, pEarleyGate202-YC, and pEarleyGate101 using the LR reaction following the manufacturer's instructions to yield C-terminal YN, C-terminal YC, and C-terminal YFP fusion constructs, respectively. All plasmids generated in this study were verified by sequencing.

\subsection{Protein Interaction as Determined by $\mathrm{Y} 2 \mathrm{H}$ and BiFC Assays}

For the Y2H assay, the Matchmaker Gold Yeast Two-Hybrid System (Clontech, Mountain View, CA, USA) was used according to the manufacturer's protocols. The activation domain (AD) vector pGADT7 and the GAL4 DNA-binding domain (BD) vector pGBKT7 were used. The prey vector pGADT7 and bait vector pGBKT7 harboring the genes to be tested were co-transformed pairwise into the yeast (Saccharomyces cerevisiae) strain AH109. $\mathrm{SD} /-\operatorname{Trp} /$-Leu (DDO) agar plates and SD/-Trp/-Leu/-His/-Ade (QDO) agar plates were used for the yeast cultures. Cells were spread on DDO plates and incubated at $30{ }^{\circ} \mathrm{C}$ for 3-5 days after transformation. Colonies grown on DDO plates were suspended in a DDO liquid medium to an $\mathrm{OD}_{600}$ of 0.6 . A $10 \times$ dilution series of $5 \mu \mathrm{L}$ aliquots of co-transformed AH109, which were spotted onto DDO or QDO agar plates supplemented with 5-Bromo4-chloro-3-indolyl- $\alpha$-D-galactopyranoside $(\mathrm{X}-\alpha-\mathrm{Gal})$ to test the expression of the MEL1 marker. The plates were incubated at $30{ }^{\circ} \mathrm{C}$ for $3-5$ days. Additionally, pGADT7-T and pGBKT7-53 interacted with the $\mathrm{Y} 2 \mathrm{H}$ assays and were used as positive controls. Moreover, pGADT7-T and pGBKT7-Lam did not form complexes and were used as negative controls. For the competitive $\mathrm{Y} 2 \mathrm{H}$ assays, co-transformed yeast cells were harvested from DDO agar 
plates and shaken in YDPA at $30^{\circ} \mathrm{C}$ for $12 \mathrm{~h}$. Then, the $\mathrm{OD}_{600}$ was adjusted to 1.0 and the culture was diluted at a ratio of 1:1000 in a QDO liquid medium at $30^{\circ} \mathrm{C}$ for $12 \mathrm{~h}$. The absorbance was measured at $\mathrm{OD}_{600}$ to quantify the interaction strength.

For the BiFC assay, two YFP fusion constructs were transformed into A. tumefaciens GV3101. Agrobacterium cultures were grown to an optical density of 1.0 at $600 \mathrm{~nm}\left(\mathrm{OD}_{600}\right)$. Equal volumes of each culture were mixed and infiltrated in $N$. benthamiana leaves using needleless syringes. The agroinfiltrated plants were maintained under normal growth conditions for 48 to $72 \mathrm{~h}$.

\subsection{Transient Expression}

The plasmids were transformed into A. tumefaciens GV3101. The transformed GV3101 was agroinfiltrated into the leaves of N. benthamiana using a needleless syringe. Agrobacteria were cultured overnight in a Luria-Bertani medium containing the appropriate antibiotics and collected by centrifugation. Then, the agrobacteria were resuspended in $10 \mathrm{mM} \mathrm{MgCl} 2$ containing $100 \mathrm{mM}$ acetosyringone. The culture was incubated for $2-3 \mathrm{~h}$ at room temperature and then diluted to an $\mathrm{OD}_{600}$ of $0.2-0.5$. N. benthamiana plants were agroinfiltrated with agrobacterial cultures, and the agroinfiltrated plants were maintained under normal growth conditions for 48 to $72 \mathrm{~h}$. A diamidine phenylindole (DAPI) stock solution $(1.0 \mathrm{mg} / \mathrm{mL})$ was prepared and stored at $-20{ }^{\circ} \mathrm{C}$. A DAPI stock solution was diluted 1:1000 in double-distilled $\mathrm{H}_{2} \mathrm{O}$ to prepare the working solution. The $\mathrm{N}$. benthamiana leaves were incubated in the working solution for 10-20 $\mathrm{min}$ at room temperature to label the nucleus.

\subsection{Confocal Microscopy}

Agroinfiltrated leaf sections were imaged at room temperature using a Leica SP8 X inverted confocal microscope with an argon laser (Leica, Wetzlar, Germany). DAPI was excited at 340-488 nm, and the emitted light was captured at 505-555 nm. GFP was excited at $488 \mathrm{~nm}$, and the emitted light was captured at 505-555 nm. YFP was excited at $514 \mathrm{~nm}$, and the emitted light was captured at 530-590 nm. Images were captured digitally and processed using the Leica Application Suite Advanced Fluorescence Lite software (LAS AF; version 2.6.3; build 8173).

\subsection{Multiple Sequence Alignment}

The amino acid sequences of eIF4Es and nCBPs from other plant species were retrieved from the NCBI (https: / / www.ncbi.nlm.nih.gov / , accessed on 28 February 2021). The DNAman 6.0 software and ClustalW2 program were used for the visualization and editing of the aligned sequences. The MEGA 7.0 software was used to construct the phylogenetic tree.

\section{Results}

\subsection{Cloning of SceIF4Ea, SceIF4Eb, SceIF(iso)4E, and ScnCBP from Sugarcane}

Four eIF4E homologs were cloned from sugarcane cultivar ROC22 based on homologous cloning. The ORFs of SceIF4Ea, SceIF4Eb, SceIF(iso)4E, and ScnCBP are 603, 567, 621, and $690 \mathrm{bp}$, respectively, with the corresponding GenBank accession numbers of MW547070, MW547071, MW547072, and KX757019. SceIF4Ea is almost identical to SceIF4Eb, with only a few amino acid differences in the N-terminus. The deduced amino acid sequences of the four SceIF4E homologs were compared with homologs from Arabidopsis thaliana, Triticum aestivum, Zea mays, Oryza sativa, and Glycine max. The results showed that the amino acid residues Trp, Phe, and His in the four SceIF4E homologs showed the distinctive pattern of eIF4E family: $\mathrm{H}\left(\mathrm{x}_{5}\right) \mathrm{W}\left(\mathrm{x}_{2}\right) \mathrm{W}\left(\mathrm{x}_{8-12}\right) \mathrm{W}\left(\mathrm{x}_{9}\right) \mathrm{F}\left(\mathrm{x}_{5}\right) \mathrm{FW}\left(\mathrm{x}_{20}\right) \mathrm{F}\left(\mathrm{x}_{7}\right) \mathrm{W}\left(\mathrm{x}_{10}\right) \mathrm{W}\left(\mathrm{x}_{12-15}\right) \mathrm{W}\left(\mathrm{x}_{34-35}\right) \mathrm{W}\left(\mathrm{x}_{31-33}\right) \mathrm{H}(\mathrm{X}$ stands for an arbitrary amino acid) (Figure 1), as described by Joshi et al. [63]. 
representing sequences from monocots or dicots, and different groups were formed by $\mathrm{C} 4$ and C3 monocot plants (Figure 2).

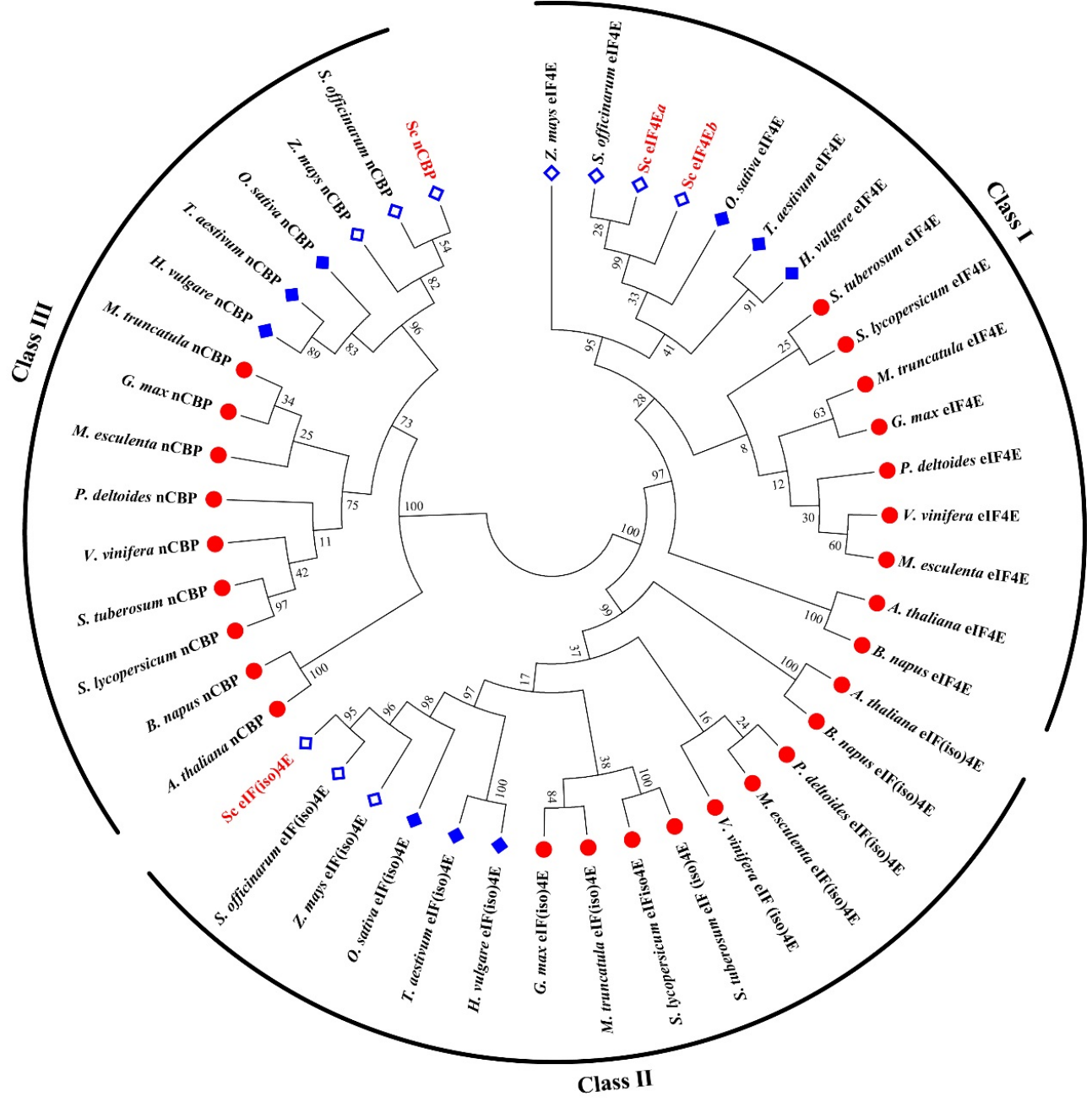

Figure 2. Phylogenetic tree analysis of eIF4E, eIF(iso)4E, and nCBP proteins from different plant species. Protein sequences were retrieved from NCBI and the accession numbers are as follows. Arabidopsis thaliana: eIF4E (AT4G18040), eIF(iso)4E (AT5G35620), nCBP (AT5G18110); Triticum aestivum: eIF4E (CAA78262), eIF(iso)4E (AAA34296), nCBP (XM.037569593); Zea mays: eIF4E (AF076954), eIF(iso)4E (CD527566), nCBP (EU959765); Vitis vinifera: eIF4E (BM437772), eIF(iso)4E (CB918946), nCBP (BM437090); Solanum tuberosum: eIF4E (BI178346), eIF(iso)4E (BG598942), nCBP (CK269484); Saccharum officinarum: eIF4E (CA248584), eIF(iso)4E (CA120131), nCBP (CA241493); Populus deltoides: eIF4E (CX176909), eIF(iso)4E (CV130917), nCBP (CX173803); Oryza sativa: eIF4E (U34597), eIF(iso)4E (U34598), nCBP (CF328046); Medicago truncatula: eIF4E (AJ502732), eIF(iso)4E (AL378243), nCBP (AL381239); Hordeum vulgare: eIF4E (CA008276), eIF(iso)4E (BU987334), nCBP (BQ467551); Glycine max: eIF4E (BI785638), eIF(iso)4E (BG045933), nCBP (BE474869); Brassica napus: eIF4E (CD814531), eIF(iso)4E (CD826731), nCBP (CD842187); Solanum lycopersicum: eIF4E (Solyc03g005870), eIF(iso)4E (Solyc09g090580), nCBP (Solyc10g080660); Manihot esculenta: eIF4E (MANES.17G063100), eIF(iso)4E (MANES.03G160000), nCBP (MANES.09G140300); Saccharum spp. hybrid ROC22: SceIF4Ea (MW547070), SceIF4Eb (MW547071), SceIF(iso)4E (MW547073), ScnCBP (KX757019), which were highlighted by the "leaves" in red. The red circle indicated the dicotyledons. The blue diamond indicated the monocotyledons with the hollow diamond for C4 plants, while the solid core of diamond for C3 plants. 


\subsection{Expression Profiles of SceIF4Ea, SceIF4Eb, SceIF(iso)4E, and ScnCBP Upon the SCMV Challenge}

The transcriptional expression profile of the four SceIF4E homologs in sugarcane cultivar ROC22 plantlets challenged by SCMV was investigated by RT-qPCR. The expression of the $C P$ gene of SCMV increased with the infection progression (Figure 3), indicating the replication of the SCMV genome and successful infection of SCMV. As the high similarity of SceIF4E $a$ and SceIF4Eb made differences difficult to be distinguished by RT-qPCR, their expression profiles were combined using one pair of primers. The results showed that the expression levels of SceIF4Ea/b, SceIF(iso) $4 E$, and ScnCBP were upregulated upon infection and then downregulated to the level of the control or slightly higher than the control level (Figure 3), which is similar to the soybean response to the Soybean mosaic virus infection [54].

A

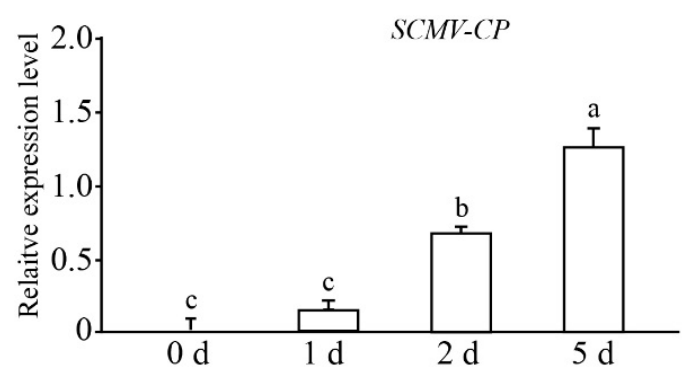

$\mathrm{C}$

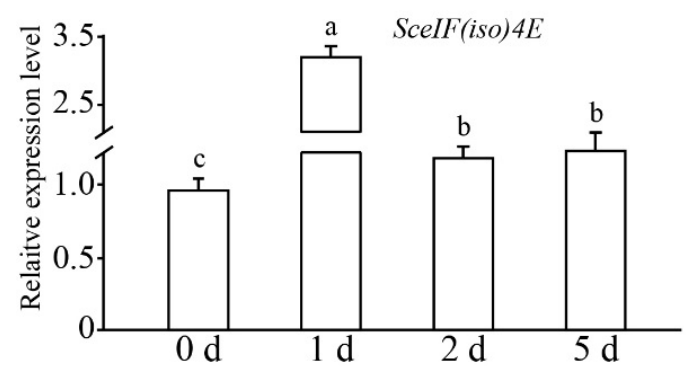

B

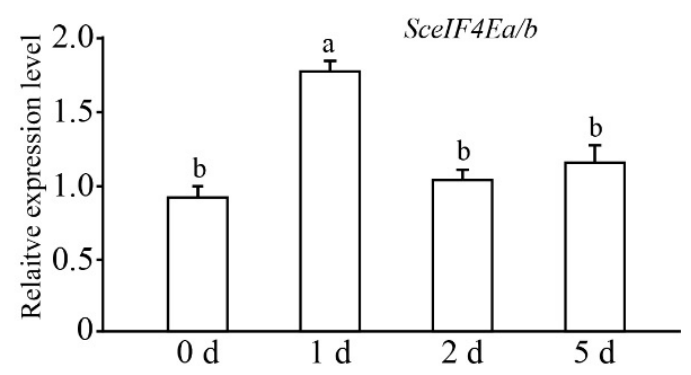

$\mathrm{D}$

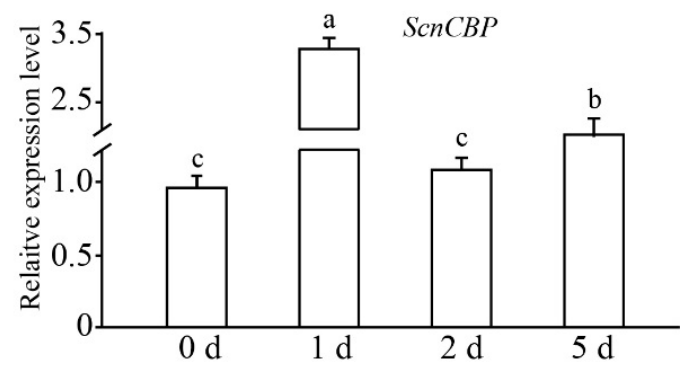

Figure 3. The expression profiles of SceIF4Ea/b, SceIF(iso) $4 E$, and ScnCBP of sugarcane cultivar ROC22 challenged by Sugarcane mosaic virus (SCMV). Leaves of ROC22 plantlets were inoculated with SCMV and sampled at different time points. Mock inoculated plants with $0.01 \mathrm{M}$ phosphate buffer $(\mathrm{pH} 7.0)$ were used as the negative controls. The $\mathrm{Y}$ axes indicates the relative expression of SCMV-CP (A), SceIF4Ea/b (B), SceIF(iso) $4 E(\mathbf{C})$, and $\operatorname{ScnCBP}(\mathbf{D})$ at $0,1,2$, and 5 days post-inoculation. The $\mathrm{X}$ axes indicates the time point of material collection. Error bars indicate $\mathrm{SD}(n=3), \mathrm{a}, \mathrm{b}$, and $\mathrm{c}$ indicate significance at the corresponding time points, $t$-test, $p<0.05$. Results were representative of three independent experiments.

\subsection{Subcellular Localization of SceIF4Ea, SceIF4Eb, SceIF(iso)4E, and ScnCBP}

To determine the subcellular localization of the four SceIF4E homologs, SceIF4EaYFP, SceIF4Eb-YFP, SceIF(iso)4E-YFP, and ScnCBP-YFP were individually expressed in N. benthamiana leaves by agroinfiltration. The leaves were infiltrated with DAPI and imaged by confocal laser scanning microscopy. YFP fluorescence was distributed in the cytoplasm and overlapped the fluorescence of DAPI in the nucleus (Figure 4), indicating that SceIF4Ea, SceIF4Eb, SceIF(iso)4E, and ScnCBP localized to the cytoplasm and nucleus. 


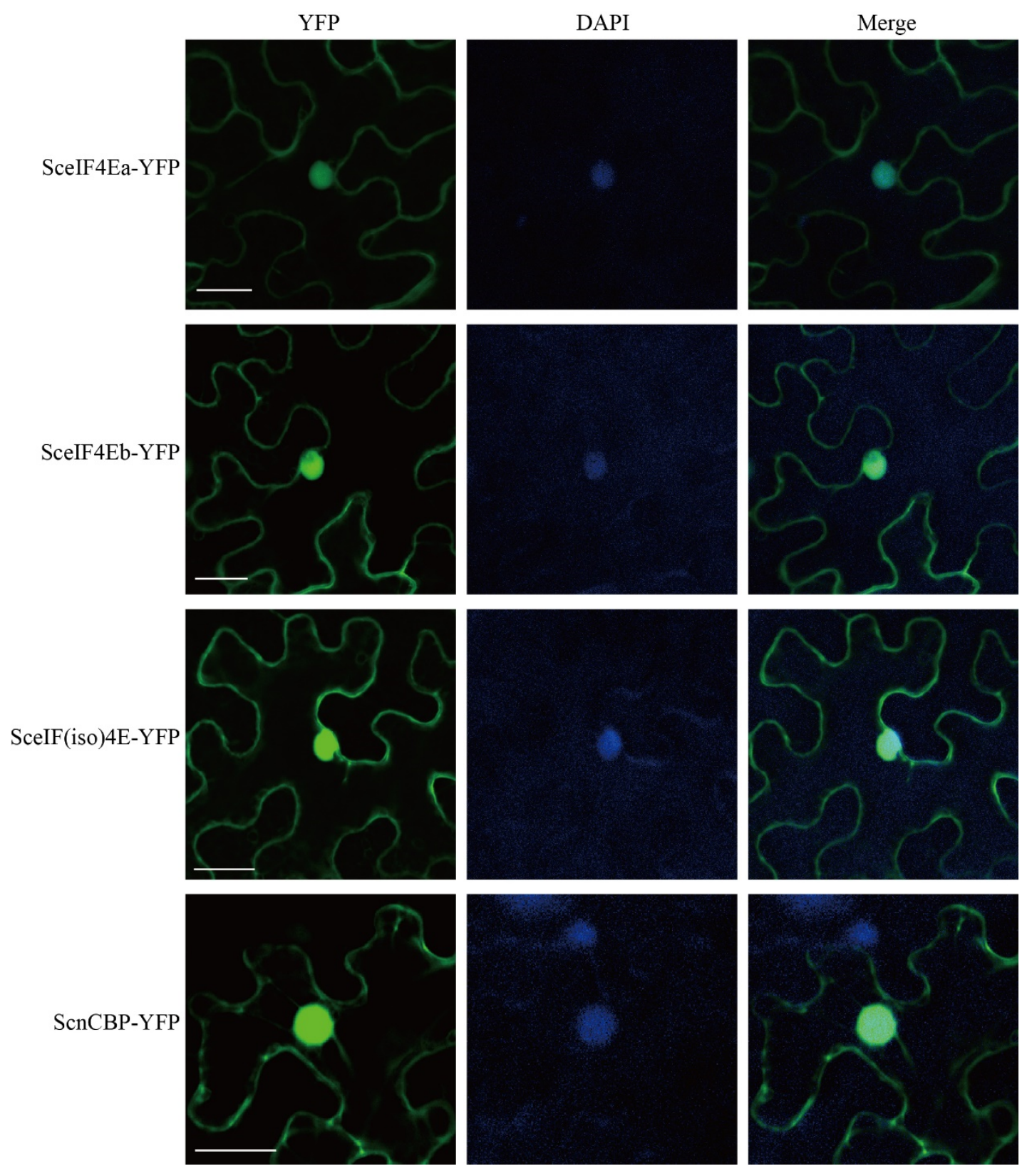

Figure 4. Subcellular localization of SceIF4Ea, SceIF4Eb, SceIF(iso)4E, and ScnCBP in Nicotiana benthamiana leaf epidermal cells. Fluorescence of YFP or YFP fusion proteins was detected by $48 \mathrm{~h}$ post agroinfiltration. The nucleus was displayed by diamidine phenylindole (DAPI) staining. Bars $=25 \mu \mathrm{m}$.

3.4. Interaction of SceIF4Ea, SceIF4Eb, SceIF(iso) $4 E$, and ScnCBP with $S C M V-/ S r M V-/ S C S M V-V P g$

Y2H based on GAL4 was applied to determine the interaction of SCMV-/SrMV-/SCSMVVPg with the four SceIF4E homologs. The bait vectors pGBKT7-SCMV-VPg, pGBKT7-SrMV$\mathrm{VPg}$, and pGBKT7-SCSMV-VPg were individually co-transformed with the prey vectors pGADT7-SceIF4Ea, pGADT7-SceIF4Eb, pGADT7-SceIF(iso)4E, and pGADT7-ScnCBP into the Saccharomyces cerevisiae strain AH109. Similar to the yeast cells co-transformed with the positive control plasmids pGBKT7-53 and pGADT7-T, the yeast cells transformed with the combination of pGBKT7-SCMV-VPg and pGADT7-SceIF4Ea, pGBKT7-SCMV-VPg and pGADT7-SceIF4Eb, pGBKT7-SCMV-VPg and pGADT7-SceIF(iso)4E, pGBKT7-SrMV-VPg and pGADT7-SceIF4Eb, pGBKT7-SrMV-VPg and pGADT7-SceIF(iso)4E, pGBKT7-SCSMV-VPg and pGADT7-SceIF(iso)4E produced blue colonies on the DDO culture medium and blue colonies on the QDO culture medium supplemented with X- $\alpha-G a l$, while other combinations 
showed no interaction, similar to the negative control plasmids pGBKT7-Lam and pGADT7$\mathrm{T}$ (Figure 5). However, the yeast cells that co-transformed with pGBKT7-SCMV-VPg and pGADT7-SceIF(iso)4E did not grow well, indicating the weak interaction between SCMV-VPg and SceIF(iso)4E (Figure 5).



Figure 5. Investigation of protein interaction by yeast two-hybrid assays. The coding sequences of SceIF4Ea, SceIF4Eb, SceIF(iso)4E, and ScnCBP were individually infused into the prey vector pGADT7 and pairwise co-transformed with the vector pGBKT7-SCMV-VPg, pGBKT7-SrMV-VPg, pGBKT7-SCSMV-VPg into the yeast AH109 cells in a $10 \times$ dilution series of $10-\mu \mathrm{L}$ aliquots, which were then plated on a non-selective medium SD/-Leu/-Trp or quadruple dropout medium $\mathrm{SD} /$-Leu/-Trp/-His/-Ade supplemented with X- $\alpha$-Gal. Yeast cells co-transformed with pGBKT7-53 and pGADT7-T were used as a positive control, pGBKT7-Lam and pGADT7-T were used as a negative control.

To further test the interactions of SCMV-/SrMV-/SCSMV-VPg with the four SceIF4E homologs in planta, BiFC assays were conducted with $N$. benthamiana leaves. The fusion constructs SCMV-VPg-YC, SrMV-VPg-YC, and SCSMV-VPg-YC were individually cotransformed with SceIF4Ea-YN, SceIF4Eb-YN, SceIF(iso)4E-YN or ScnCBP-YN into Agrobacterium tumefaciens GV3101 and then agroinfiltrated into N. benthamiana leaves. Within $48 \mathrm{hpa}$, the yellow fluorescence of YFP was observed by confocal microscopy. The results of the BiFC assays confirmed the interaction between SCMV-/SrMV-/SCSMV-VPg and SceIF4Ea, SceIF4Eb, SceIF(iso)4E or ScnCBP, as demonstrated by the $\mathrm{Y} 2 \mathrm{H}$ assays, with the exception that ScnCBP interacted with SCMV-/SrMV-/SCSMV-VPg (Figure 6). As expected, interactions with the positive controls SCMV-VPg-YN and ScELC-YC were observed [57], while the negative controls emitted no fluorescence signals (Figure 6). 


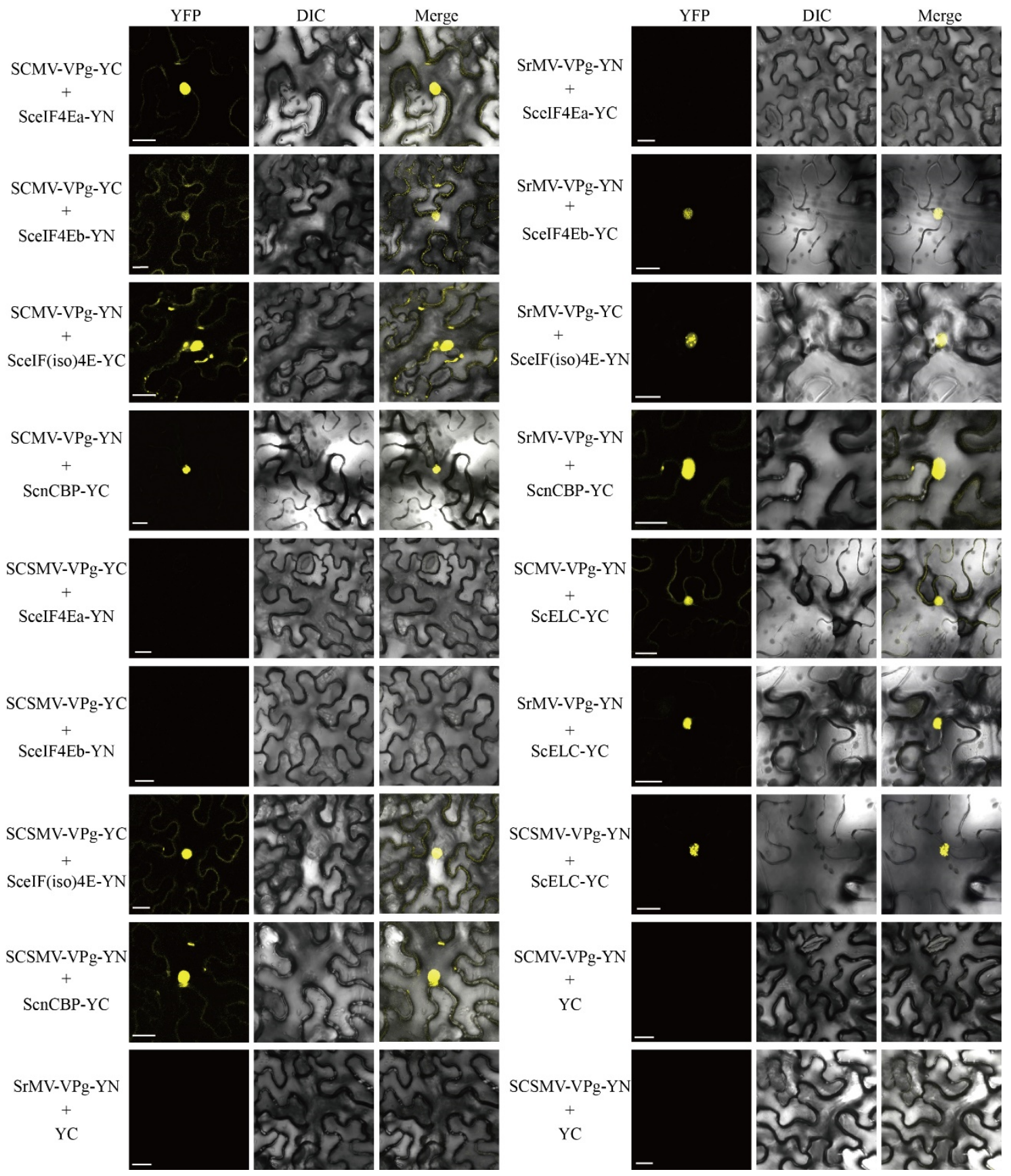

Figure 6. Investigation of protein interaction by bimolecular fluorescence complementation assays. Agrobacteria harboring YC/YN fusion proteins were co-infiltrated into Nicotiana benthamiana leaves, respectively. The leaf epidermal cells pairwise co-transformed with SCMV-VPg-YN, SrMV-VPg-YN or SCSMV-VPg-YN and ScELC-YC were used as positive controls, while SCMV-VPg-YN, SrMV-VPg-YN or SCSMV-VPg-YN and YC were used as negative controls. The images were captured at $48 \mathrm{~h}$ post agroinfiltration. Bars $=25 \mu \mathrm{m}$.

\subsection{Competitive Interaction of SceIF4Ea, SceIF4Eb, SceIF(iso) 4 E with} $S C M V-/ S r M V-/ S C S M V-V P g$

Competitive $\mathrm{Y} 2 \mathrm{H}$ assays were performed to investigate the interaction of SceIF4Ea, SceIF4Eb, and SceIF(iso)4E with SCMV-/SrMV-/SCSMV-VPg. Interacting proteins were inserted into the bridge vector and used as competitors. The interacting proteins were constructed into AD or BD vectors and pairwise co-transformed with the competitor or an empty pBridge vector as a negative control into the $S$. cerevisiae strain AH109 cultured on a QDO agar medium. Yeast cells co-transformed with the competitor or an empty 
pBridge vector were cultured in a QDO liquid medium. The QDO liquid medium was also used to quantify the growth of the transformed yeast cells by monitoring the $\mathrm{OD}_{600}$ value. As SceIF(iso)4E interacted with SCMV-VPg, SrMV-VPg, and SCSMV-VPg, each VPg was expressed via a pBridge vector to investigate the interaction of other VPgs with SceIF(iso)4E. Compared with the controls, the yeast cells showed no obvious difference, indicating that SCMV-VPg, SrMV-VPg, and SCSMV-VPg did not compete with each other for the interaction with SceIF(iso)4E (Figure 7), as demonstrated by QDO liquid medium culture assays (Figure S1a-c). As SrMV-VPg interacted with SceIF4Eb or SceIF(iso)4E, SceIF4Eb or SceIF(iso)4E was individually constructed into a pBridge vector to investigate the interaction of SrMV-VPg with eIF4Eb or SceIF(iso)4E, respectively. The results showed that SceIF(iso)4E interfered with the interaction between SceIF4Eb and SrMV-VPg, while SceIF4Eb did not interfere with the interaction between SceIF(iso)4E and SrMVVPg (Figure 7; Figure S1d), indicating that SceIF(iso)4E is preferentially utilized by SrMV. For the interaction of SCMV-VPg with SceIF4Eb or SceIF(iso)4E, the results showed that SceIF4Eb did not interfere with the interaction between SCMV-VPg and SceIF(iso)4E, while SceIF(iso)4E did not interfere with the interaction between SCMV-VPg and SceIF4Eb, which was confirmed by QDO liquid medium culture assays (Figure 7; Figure S1e).

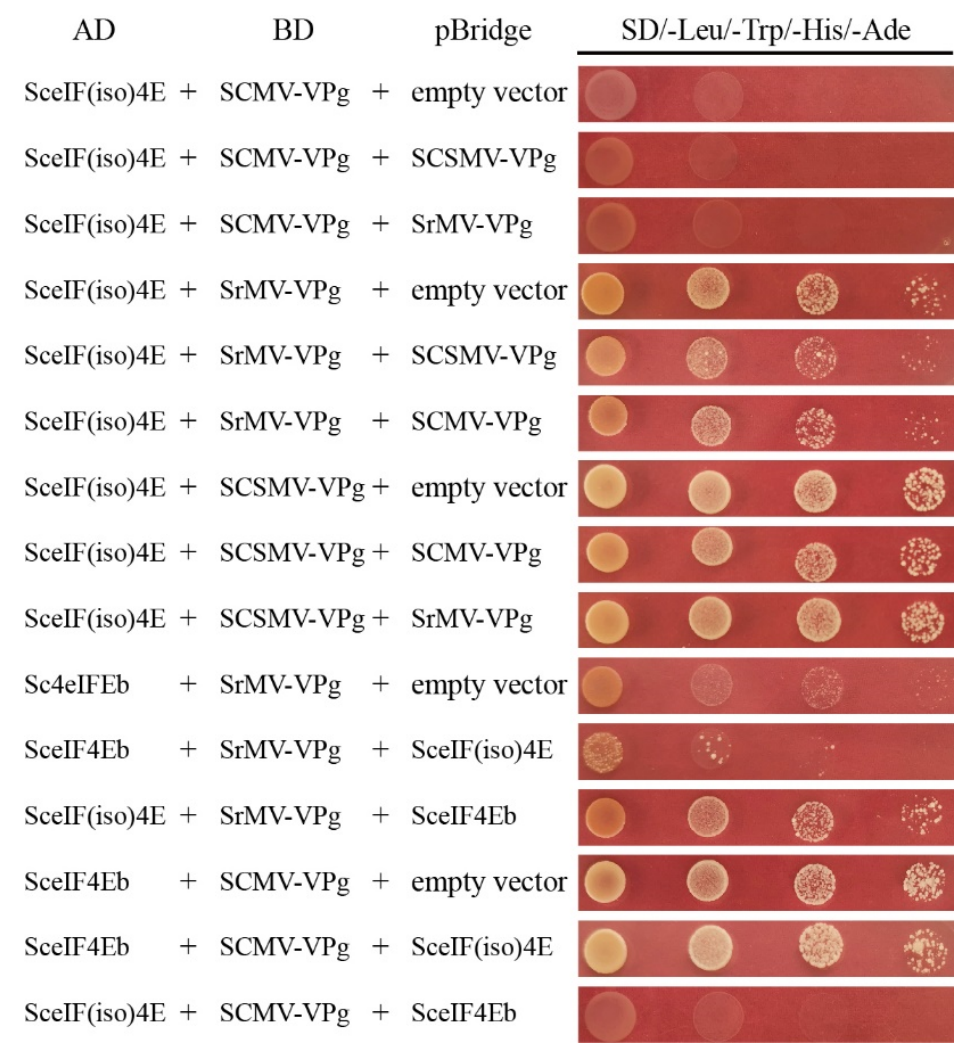

Figure 7. Competitive yeast two-hybrid assays. SCMV-VPg, SrMV-VPg, SCSMV-VPg, eIF4Eb, eIF(iso)4E were used as the competitor to test the interaction of SCMV-VPg, SrMV-VPg, SCSMV-VPg with eIF4Eb, eIF(iso) $4 \mathrm{E}$, respectively. The competitor was co-transformed with the paired interacting proteins fused with the activation domain (AD) or DNA-binding domain (BD) into the yeast AH109 cells in a $10 \times$ dilution series of $10-\mu \mathrm{L}$ aliquots, which were then plated on the quadruple dropout medium SD/-Leu/-Trp/-His/-Ade.

\section{Discussion}

In the present study, the coding genes of SceIF4Ea, SceIF4Eb, SceIF(iso)4E, and ScnCBP were cloned from susceptible sugarcane cultivar ROC22, and their interaction with the VPgs of SCMV, SrMV or SCSMV was investigated. This study indicated that SceIF4Ea, SceIF4Eb, and SceIF(iso)4E were selectively employed by SCMV, SrMV or SCSMV. Two eIF4Es were 
identified, i.e., SceIF4Ea and SceIF4Eb, whose sequences were similar, with only few amino acid residues differences at the N-terminus (Figure 2). Intriguingly, SceIF4Ea and SceIF4Eb interacted with SCMV-VPg, whereas SceIF4Eb interacted with SrMV-VPg, but SceIF4Ea did not (Figures 5 and 6), which indicated that the N-terminus of eIF4E is involved in the interaction with potyviral VPg. It might deepen the understanding of the molecular mechanism of VPg interaction with eIF4E to identify the key residue in the N-terminus of $\mathrm{SceIF} 4 \mathrm{~Eb}$ that mediates the interaction with SrMV-VPg.

ScnCBP did not interact with the VPgs of SCMV, SrMV or SCSMV in the Y2H assays, but the BiFC assays with the $N$. benthamiana leaves yielded positive results (Figure 5). In A. thaliana, nCBP showed affinity to the cap structure comparable to that shown by eIF(iso)4E [64]. The cap binding complex comprises many components [65,66]. Considering that ScnCBP was upregulated upon SCMV infection (Figure 3), we speculate that other cap binding components mediate the interaction of ScnCBP with the VPgs of SCMV, SrMV or SCSMV in vivo. The nCBP mediated recessive resistance against viruses in the Alphaflexiviridae and Betaflexiviridae families [67]. Simultaneous CRISPR/Cas9-mediated editing of two nCBP alleles reduced the cassava brown streak disease symptom severity and incidence caused by Cassava brown streak virus and Ugandan cassava brown streak virus in the Potyviridae family [68]. Therefore, it is reasonable to conclude that ScnCBP plays roles in the response to the mosaic virus infection of sugarcane.

SCMV-VPg, SrMV-VPg, and SCSMV-VPg interacted and did not compete with each other for the interaction with SceIF(iso)4E (Figures 5-7), indicating that SceIF(iso)4E was extensively employed by SCMV, SrMV, and SCSMV. Specifically, SCSMV-VPg was found to selectively interact with SceIF(iso)4E (Figures 5 and 6), making SceIF(iso) $4 E$ an eminent target for the molecular breeding of sugarcane plants with resistance to SCSMV. As SceIF(iso)4E was preferentially employed by SrMV (Figure 7 and Figure S1), mutation of SceIF(iso) $4 E$ might force SrMV to utilize SceIF4Eb with suboptimal binding affinity, thereby attenuating mosaic disease progression caused by SrMV.

Growing virus-resistant sugarcane cultivars are the best way to prevent severe losses of yield and quality [4]. However, it is difficult to combine eminent agricultural traits with mosaic resistance due to the complicated sugarcane genome [60]. With the development of RNAi and the increasing regeneration efficiency of sugarcane calli, the $C P$ gene was utilized to create resistant sugarcane germplasm against SCMV or SrMV [17,69]. However, the $C P$ genes varied greatly in sequence [70]. Genetically modified sugarcane plants that transformed with the $\mathrm{CP}$ gene from SrMV-SCH were resistant to SrMV-SCH and the closely related SrMV-SCI and SrMV-SCM strains (95\% nucleotide similarity) but not to the more distantly related SCMV strain D (75\% nucleotide similarity) [69]. Therefore, the resistance derived from pathogens might make it difficult to create broadly resistant germplasms. As there are numerous successful reports of potyviruses management obtained by the mutation of eIF4E or its isoform [44-51] and the identification of natural recessive resistance genes against potyviruses $[37,55,56,67]$, mutation of $S c e I F(i s o) 4 E$ may confer to sugarcane plants resistance to SCSMV, while simultaneous mutation of SceIF4Eb and SceIF(iso) $4 E$ may confer to sugarcane plants broad resistance to both SrMV and SCSMV. However, this method should be tested by comprehensive experiments, as a recent study by Zafirov et al. showed that eIF4E1-knockout $A$. thaliana mutants were resistant to Clover yellow vein virus (CIYVV) but hypersusceptible to TuMV [71]. Zafirov et al. suggested that gene editing by CRISPR/Cas9 is superior to gene knockout [71-73]. Therefore, further experiments should be conducted to investigate the key amino acid residues that mediate the interaction of SceIF4Ea, SceIF4Eb, SceIF(iso)4E or ScnCBP with SCMV-VPg, SrMV-VPg or SCSMV-VPg.

Supplementary Materials: The following are available online at https:/ / www.mdpi.com/1999-491 5/13/3/518/s1, Figure S1: Yeast growth assay, Table S1: Primers used in this study.

Author Contributions: J.X., Z.Y., and F.W. conceived and designed the experiments; Z.Y., M.D., G.C., S.L., H.Z., H.S., Y.Z., F.W., and G.H. performed the experiments; J.X. and F.W. analyzed the data and 
wrote the paper; J.X. and M.Z. acquired funding. All authors have read and agreed to the published version of the manuscript.

Funding: This research was funded by the National Natural Science Foundation of China (grant number 31171605), the Science and Technology Innovation Project of Fujian Agriculture and Forestry University (grants number CXZX2017262, CXZX2019132G), and the Open Project of the State Key Laboratory for Conservation and Utilization of Subtropical Agro-bioresources (grant number SKLCUSA-b201809).

Institutional Review Board Statement: Not applicable.

Informed Consent Statement: Not applicable.

Data Availability Statement: The data that support the findings of this study are available from the corresponding author upon reasonable request.

Conflicts of Interest: The authors declare no conflict of interest. The funders had no role in the design of the study; in the collection, analyses, or interpretation of data; in the writing of the manuscript, or in the decision to publish the results.

\section{References}

1. Rae, A.; Jackson, M.; Nguyen, C.H.; Bonnett, G.D. Functional Specialization of Vacuoles in Sugarcane Leaf and Stem. Trop. Plant Biol. 2008, 2, 13-22. [CrossRef]

2. Lam, E.; Shine, J.; Da Silva, J.; Lawton, M.; Bonos, S.; Calvino, M.; Carrer, H.; Silva-Filho, M.C.; Glynn, N.; Helsel, Z.; et al. Improving sugarcane for biofuel: Engineering for an even better feedstock. GCB Bioenergy 2009, 1, 251-255. [CrossRef]

3. Sabatier, D.; Martiné, J.; Chiroleu, F.; Roussel, C.; Letourmy, P.; Antwerpen, R.V.; Gabrielle, B.; Ney, B. Optimization of sugarcane farming as a multipurpose crop for energy and food production. GCB Bioenergy 2015, 7. [CrossRef]

4. Wu, L.J.; Zu, X.F.; Wang, S.X.; Chen, Y.H. Sugarcane mosaic virus-Long history but still a threat to industry. Crop Prot. 2012, 42, 74-78. [CrossRef]

5. Zambrano, A.Y.; Demey, J.R.; Fuchs, M.; Gonzalez, V.; Rea, R.; De Sousa, O.; Gutierrez, Z. Selection of sugarcane plants resistant to SCMV. Plant Sci. 2003, 165, 221-225. [CrossRef]

6. Xie, Y.J.; Wang, M.Q.; Xu, D.L.; Li, R.H.; Zhou, G.H. Simultaneous detection and identification of four sugarcane viruses by one-step RT-PCR. J. Virol. Methods 2009, 162, 64-68. [CrossRef]

7. Yang, Z.N.; Mirkov, T.E. Sequence and Relationships of Sugarcane Mosaic and Sorghum Mosaic Virus Strains and Development of RT-PCR-Based RFLPs for Strain Discrimination. Phytopathology 1997, 87, 932-939. [CrossRef]

8. Grisham, M.P.; Pan, Y.B. A genetic shift in the virus strains that cause mosaic in Louisiana sugarcane. Plant Dis. 2007, 91, 453-458. [CrossRef]

9. Perera, M.F.; Filippone, M.P.; Ramallo, C.J.; Cuenya, M.I.; Garcia, M.L.; Ploper, L.D.; Castagnaro, A.P. Genetic Diversity Among Viruses Associated with Sugarcane Mosaic Disease in Tucumaan, Argentina. Phytopathology 2009, 99, 38-49. [CrossRef] [PubMed]

10. Ha, C.; Revill, P.; Harding, R.M.; Vu, M.; Dale, J.L. Identification and sequence analysis of potyviruses infecting crops in Vietnam. Arch. Virol. 2008, 153, 45-60. [CrossRef]

11. Li, W.F.; Wang, X.Y.; Huang, Y.K.; Shan, H.L.; Luo, Z.M.; Ying, X.M.; Zhang, R.Y.; Shen, K.; Yin, J. Screening sugarcane germplasm resistant to Sorghum mosaic virus. Crop Prot. 2013, 43, 27-30. [CrossRef]

12. Li, W.F.; Shan, H.L.; Zhang, R.Y.; Wang, X.Y.; Yang, K.; Luo, Z.M.; Yin, J.; Cang, X.Y.; Li, J.; Huang, Y.K. Identification of resistance to Sugarcane streak mosaic virus (SCSMV) and Sorghum mosaic virus (SrMV) in new elite sugarcane varieties/clones in China. Crop Prot. 2018, 110, 77-82. [CrossRef]

13. Li, W.F.; Shan, H.L.; Cang, X.Y.; Lu, X.; Zhang, R.Y.; Wang, X.Y.; Yin, J.; Luo, Z.M.; Huang, Y.K. Identification and Evaluation of Resistance to Sugarcane Streak Mosaic Virus (SCSMV) and Sorghum Mosaic Virus (SrMV) in Excellent Sugarcane Innovation Germplasms in China. Sugar Tech 2019, 21, 481-485. [CrossRef]

14. Filloux, D.; Fernandez, E.; Comstock, J.C.; Mollov, D.; Roumagnac, P.; Rott, P. Viral Metagenomic-Based Screening of Sugarcane from Florida Reveals Occurrence of Six Sugarcane-Infecting Viruses and High Prevalence of Sugarcane yellow leaf virus. Plant Dis. 2018, 102, 2317-2323. [CrossRef] [PubMed]

15. Akbar, S.; Yao, W.; Yu, K.; Qin, L.F.; Ruan, M.H.; Powell, C.A.; Chen, B.S.; Zhang, M.Q. Photosynthetic characterization and expression profiles of sugarcane infected by Sugarcane mosaic virus (SCMV). Photosynth. Res. 2020. [CrossRef]

16. Putra, L.K.; Kristini, A.; Achadian, E.M.; Damayanti, T.A. Sugarcane streak mosaic virus in Indonesia: Distribution, Characterisation, Yield Losses and Management Approaches. Sugar Tech 2014, 16, 392-399. [CrossRef]

17. Yao, W.; Ruan, M.; Qin, L.; Yang, C.; Chen, R.; Chen, B.; Zhang, M. Field Performance of Transgenic Sugarcane Lines Resistant to Sugarcane Mosaic Virus. Front. Plant Sci. 2017, 8, 104. [CrossRef]

18. Ricaud, C.; Egan, B.; Gillaspie, A.G.; Hughes, C.G. Diseases of Sugarcane: Major Diseases; Elsevier: New York, NY, USA, 1989; pp. 301-322.

19. Charron, C.; Nicolai, M.; Gallois, J.L.; Robaglia, C.; Moury, B.T.; Palloix, A.; Caranta, C. Natural variation and functional analyses provide evidence for co-evolution between plant eIF4E and potyviral VPg. Plant J. 2008, 54, 56-68. [CrossRef] 
20. Ward, C.; Shukla, D.J.I. Taxonomy of potyviruses: Current problems and some solutions. Intervirology 1991, 32 5, $269-296$. [CrossRef]

21. Hema, M.; Sreenivasulu, P.; Savithri, H.S. Taxonomic position of Sugarcane streak mosaic virus in the family Potyviridae. Arch Virol. 2002, 147, 1997-2007. [CrossRef]

22. Xu, D.L.; Zhou, G.H.; Xie, Y.J.; Mock, R.; Li, R. Complete nucleotide sequence and taxonomy of Sugarcane streak mosaic virus, member of a novel genus in the family Potyviridae. Virus Genes 2010, 40, 432-439. [CrossRef]

23. Li, W.; He, Z.; Li, S.; Huang, Y.; Zhang, Z.; Jiang, D.; Wang, X.; Luo, Z. Molecular characterization of a new strain of Sugarcane streak mosaic virus (SCSMV). Arch Virol. 2011, 156, 2101-2104. [CrossRef] [PubMed]

24. Urcuqui-Inchima, S.; Haenni, A.L.; Bernardi, F. Potyvirus proteins: A wealth of functions. Virus Res. 2001, 74, 157-175. [CrossRef]

25. Wang, A.M. Dissecting the Molecular Network of Virus-Plant Interactions: The Complex Roles of Host Factors. Annu. Rev. Phytopathol. 2015, 53, 45-66. [CrossRef] [PubMed]

26. Olspert, A.; Chung, B.Y.; Atkins, J.F.; Carr, J.P.; Firth, A.E. Transcriptional slippage in the positive-sense RNA virus family Potyviridae. EMBO Rep. 2015, 16, 995-1004. [CrossRef] [PubMed]

27. Olspert, A.; Carr, J.P.; Firth, A.E. Mutational analysis of the Potyviridae transcriptional slippage site utilized for expression of the P3N-PIPO and P1N-PISPO proteins. Nucleic Acids Res. 2016, 44, 7618-7629. [CrossRef]

28. Riechmann, J.L.; Laín, S.; García, J.A. Highlights and prospects of potyvirus molecular biology. J. Gen. Virol. 1992, 73 Pt 1, 1-16. [CrossRef]

29. Cui, X.; Yaghmaiean, H.; Wu, G.; Wu, X.; Chen, X.; Thorn, G.; Wang, A. The C-terminal region of the Turnip mosaic virus P3 protein is essential for viral infection via targeting P3 to the viral replication complex. Virology 2017, 510, 147-155. [CrossRef]

30. Cheng, G.; Dong, M.; Xu, Q.; Peng, L.; Yang, Z.; Wei, T.; Xu, J. Dissecting the Molecular Mechanism of the Subcellular Localization and Cell-to-cell Movement of the Sugarcane mosaic virus P3N-PIPO. Sci. Rep. 2017, 7, 9868. [CrossRef]

31. Cheng, G.; Yang, Z.; Zhang, H.; Zhang, J.; Xu, J. Remorin interacting with PCaP1 impairs Turnip mosaic virus intercellular movement but is antagonised by VPg. New Phytol. 2020, 225, 2122-2139. [CrossRef]

32. Chai, M.; Wu, X.; Liu, J.; Fang, Y.; Luan, Y.; Cui, X.; Zhou, X.; Wang, A.; Cheng, X. P3N-PIPO Interacts with P3 via the Shared N-Terminal Domain to Recruit Viral Replication Vesicles for Cell-to-Cell Movement. J. Virol. 2020, 94. [CrossRef] [PubMed]

33. Gingras, A.C.; Raught, B.; Sonenberg, N. eIF4 initiation factors: Effectors of mRNA recruitment to ribosomes and regulators of translation. Annu. Rev. Biochem. 1999, 68, 913-963. [CrossRef] [PubMed]

34. Sonenberg, N.; Hershey, J.; Mathews, M. Translational Control of Gene Expression; Cold Spring Harbor Laboratory Press: Cold Spring Harbor, NY, USA, 2000; pp. 1-31.

35. Wittmann, S.; Chatel, H.; Fortin, M.G.; Laliberté, J.F. Interaction of the viral protein genome linked of turnip mosaic potyvirus with the translational eukaryotic initiation factor (iso) 4E of Arabidopsis thaliana using the yeast two-hybrid system. Virology 1997, 234, 84-92. [CrossRef]

36. Leonard, S.; Plante, D.; Wittmann, S.; Daigneault, N.; Fortin, M.; Laliberté, J.F. Complex Formation between Potyvirus VPg and Translation Eukaryotic Initiation Factor 4E Correlates with Virus Infectivity. J. Virol. 2000, 74, 7730-7737. [CrossRef] [PubMed]

37. Wang, A.M.; Krishnaswamy, S. Eukaryotic translation initiation factor $4 \mathrm{E}$-mediated recessive resistance to plant viruses and its utility in crop improvement. Mol. Plant Pathol. 2012, 13, 795-803. [CrossRef] [PubMed]

38. Anindya, R.; Chittori, S.; Savithri, H.S. Tyrosine 66 of Pepper vein banding virus genome-linked protein is uridylylated by RNA-dependent RNA polymerase. Virology 2005, 336, 154-162. [CrossRef] [PubMed]

39. Belliot, G.; Sosnovtsev, S.V.; Chang, K.-O.; McPhie, P.; Green, K.Y. Nucleotidylylation of the VPg protein of a human norovirus by its proteinase-polymerase precursor protein. Virology 2008, 374, 33-49. [CrossRef]

40. Murphy, J.F.; Rychlik, W.; Rhoads, R.E.; Hunt, A.G.; Shaw, J.G. A tyrosine residue in the small nuclear inclusion protein of tobacco vein mottling virus links the VPg to the viral RNA. J. Virol. 1991, 65, 511-513. [CrossRef] [PubMed]

41. Murphy, J.; Klein, P.G.; Hunt, A.; Shaw, J.J.V. Replacement of the tyrosine residue that links a potyviral VPg to the viral RNA is lethal. Virology 1996, 220 2, 535-538. [CrossRef]

42. Robaglia, C.; Caranta, C. Translation initiation factors: A weak link in plant RNA virus infection. Trends Plant Sci. 2006, 11, 40-45. [CrossRef]

43. Goodfellow, I. The genome-linked protein VPg of vertebrate viruses-A multifaceted protein. Curr. Opin. Virol. 2011, 1, 355-362. [CrossRef]

44. Lellis, A.D.; Kasschau, K.D.; Whitham, S.A.; Carrington, J.C. Loss-of-susceptibility mutants of Arabidopsis thaliana reveal an essential role for eIF(iso)4E during potyvirus infection. Curr. Biol. 2002, 12, 1046-1051. [CrossRef]

45. Duprat, A.; Caranta, C.; Revers, F.; Menand, B.; Browning, K.S.; Robaglia, C. The Arabidopsis eukaryotic initiation factor (iso)4E is dispensable for plant growth but required for susceptibility to potyviruses. Plant J. 2002, 32, 927-934. [CrossRef]

46. Sato, M.; Nakahara, K.; Yoshii, M.; Ishikawa, M.; Uyeda, I. Selective involvement of members of the eukaryotic initiation factor 4E family in the infection of Arabidopsis thaliana by potyviruses. FEBS Lett. 2005, 579, 1167-1171. [CrossRef] [PubMed]

47. Masuta, C.; Yamana, T.; Tacahashi, Y.; Uyeda, I.; Sato, M.; Ueda, S.; Matsumura, T. Development of clover yellow vein virus as an efficient, stable gene-expression system for legume species. Plant J. 2000, 23, 539-546. [CrossRef]

48. Ruffel, S.; Dussault, M.H.; Palloix, A.; Moury, B.; Bendahmane, A.; Robaglia, C.; Caranta, C. A natural recessive resistance gene against potato virus $\mathrm{Y}$ in pepper corresponds to the eukaryotic initiation factor 4E (eIF4E). Plant J. 2002, 32, 1067-1075. [CrossRef] 
49. Nicaise, V.; German-Retana, S.; Sanjuan, R.; Dubrana, M.P.; Mazier, M.; Maisonneuve, B.; Candresse, T.; Caranta, C.; LeGall, O. The eukaryotic translation initiation factor $4 \mathrm{E}$ controls lettuce susceptibility to the Potyvirus Lettuce mosaic virus. Plant Physiol. 2003, 132, 1272-1282. [CrossRef]

50. Piron, F.; Nicolai, M.; Minoia, S.; Piednoir, E.; Moretti, A.; Salgues, A.; Zamir, D.; Caranta, C.; Bendahmane, A. An Induced Mutation in Tomato eIF4E Leads to Immunity to Two Potyviruses. PLoS ONE 2010, 5, e11313. [CrossRef]

51. Mazier, M.; Flamain, F.; Nicolai, M.; Sarnette, V.; Caranta, C. Knock-Down of Both eIF4E1 and eIF4E2 Genes Confers BroadSpectrum Resistance against Potyviruses in Tomato. PLoS ONE 2011, 6, e29595. [CrossRef] [PubMed]

52. Maule, A.J.; Caranta, C.; Boulton, M.I. Sources of natural resistance to plant viruses: Status and prospects. Mol. Plant Pathol. 2007, 8, 223-231. [CrossRef] [PubMed]

53. Yoon, Y.J.; Venkatesh, J.; Lee, J.H.; Kim, J.; Lee, H.E.; Kim, D.S.; Kang, B.C. Genome Editing of eIF4E1 in Tomato Confers Resistance to Pepper Mottle Virus. Front. Plant Sci. 2020, 11, 1098. [CrossRef]

54. Gao, L.; Luo, J.Y.; Ding, X.N.; Wang, T.; Hu, T.; Song, P.W.; Zhai, R.; Zhang, H.Y.; Zhang, K.; Li, K.; et al. Soybean RNA interference lines silenced for eIF4E show broad potyvirus resistance. Mol. Plant Pathol. 2020, 21, 303-317. [CrossRef] [PubMed]

55. Sanfacon, H. Plant Translation Factors and Virus Resistance. Viruses 2015, 7, 3392-3419. [CrossRef] [PubMed]

56. Swisher Grimm, K.D.; Porter, L.D. Development and Validation of KASP Markers for the Identification of Pea seedborne mosaic virus Pathotype P1 Resistance in Pisum sativum. Plant Dis. 2020, 104, 1824-1830. [CrossRef]

57. Zhai, Y.S.; Deng, Y.Q.; Cheng, G.Y.; Peng, L.; Zheng, Y.R.; Yang, Y.Q.; Xu, J.S. Sugarcane Elongin C is involved in infection by sugarcane mosaic disease pathogens. Biochem. Biophys. Res. Commun. 2015, 466, 312-318. [CrossRef] [PubMed]

58. Zhang, H.; Cheng, G.; Yang, Z.; Wang, T.; Xu, J. Identification of Sugarcane Host Factors Interacting with the 6K2 Protein of the Sugarcane Mosaic Virus. Int. J. Mol. Sci. 2019, 20, 3867. [CrossRef] [PubMed]

59. Xu, J.S.; Deng, Y.Q.; Cheng, G.Y.; Zhai, Y.S.; Peng, L.; Dong, M.; Xu, Q.; Yang, Y.Q. Sugarcane mosaic virus infection of model plants Brachypodium distachyon and Nicotiana benthamiana. J. Intergr. Agric. 2019, 18, 2294-2301. [CrossRef]

60. Zhang, J.S.; Zhang, X.T.; Tang, H.B.; Zhang, Q.; Hua, X.T.; Ma, X.K.; Zhu, F.; Jones, T.; Zhu, X.G.; Bowers, J.; et al. Allele-defined genome of the autopolyploid sugarcane Saccharum spontaneum L. Nat. Genet. 2018, 50. [CrossRef]

61. Iskandar, H.M.; Simpson, R.S.; Casu, R.E.; Bonnett, G.D.; Maclean, D.J.; Manners, J.M. Comparison of reference genes for quantitative real-time polymerase chain reaction analysis of gene expression in sugarcane. Plant Mol. Biol. Report. 2004, 22, 325-337. [CrossRef]

62. Ling, H.; Wu, Q.; Guo, J.; Xu, L.; Que, Y. Comprehensive selection of reference genes for gene expression normalization in sugarcane by real time quantitative RT-PCR. PLoS ONE 2014, 9, e97469. [CrossRef]

63. Joshi, B.; Lee, K.; Maeder, D.L.; Jagus, R. Phylogenetic analysis of eIF4E-family members. BMC Evol. Biol. 2005, 5, 48. [CrossRef]

64. Kropiwnicka, A.; Kuchta, K.; Lukaszewicz, M.; Kowalska, J.; Jemielity, J.; Ginalski, K.; Darzynkiewicz, E.; Zuberek, J. Five eIF4E isoforms from Arabidopsis thaliana are characterized by distinct features of cap analogs binding. Biochem. Biophys. Res. Commun. 2015, 456, 47-52. [CrossRef]

65. Gebhardt, A.; Habjan, M.; Benda, C.; Meiler, A.; Haas, D.A.; Hein, M.Y.; Mann, A.; Mann, M.; Habermann, B.; Pichlmair, A. mRNA export through an additional cap-binding complex consisting of NCBP1 and NCBP3. Nat. Commun. 2015, 6, 8192. [CrossRef]

66. Dou, Y.; Kalmykova, S.; Pashkova, M.; Oghbaie, M.; Jiang, H.; Molloy, K.R.; Chait, B.T.; Rout, M.P.; Fenyo, D.; Jensen, T.H.; et al. Affinity proteomic dissection of the human nuclear cap-binding complex interactome. Nucleic Acids Res. 2020, 48, 10456-10469. [CrossRef] [PubMed]

67. Keima, T.; Hagiwara-Komoda, Y.; Hashimoto, M.; Neriya, Y.; Koinuma, H.; Iwabuchi, N.; Nishida, S.; Yamaji, Y.; Namba, S. Deficiency of the eIF4E isoform nCBP limits the cell-to-cell movement of a plant virus encoding triple-gene-block proteins in Arabidopsis thaliana. Sci. Rep. 2017, 7, 39678. [CrossRef] [PubMed]

68. Gomez, M.A.; Lin, Z.D.; Moll, T.; Chauhan, R.D.; Hayden, L.; Renninger, K.; Beyene, G.; Taylor, N.J.; Carrington, J.C.; Staskawicz, B.J.; et al. Simultaneous CRISPR/Cas9-mediated editing of cassava eIF4E isoforms $n C B P-1$ and $n C B P-2$ reduces cassava brown streak disease symptom severity and incidence. Plant Biotechnol. J. 2019, 17, 421-434. [CrossRef]

69. Ingelbrecht, I.L.; Irvine, J.E.; Mirkov, T.E. Posttranscriptional gene silencing in transgenic sugarcane. Dissection of homologydependent virus resistance in a monocot that has a complex polyploid genome. Plant Physiol 1999, 119, 1187-1198. [CrossRef] [PubMed]

70. Li, Y.Q.; Liu, R.Y.; Zhou, T.; Fan, Z.F. Genetic diversity and population structure of Sugarcane mosaic virus. Virus Res. 2013, 171, 242-246. [CrossRef]

71. Zafirov, D.; Giovinazzo, N.; Bastet, A.; Gallois, J.-L. When a knockout is an Achilles' heel: Resistance to one potyvirus species triggers hypersusceptibility to another one in Arabidopsis thaliana. Mol. Plant Pathog. 2021, 22, 334-347. [CrossRef]

72. Gauffier, C.; Lebaron, C.; Moretti, A.; Constant, C.; Moquet, F.; Bonnet, G.; Caranta, C.; Gallois, J.L. A TILLING approach to generate broad-spectrum resistance to potyviruses in tomato is hampered by eIF4E gene redundancy. Plant J. 2016, 85, 717-729. [CrossRef]

73. Bastet, A.; Zafirov, D.; Giovinazzo, N.; Guyon-Debast, A.; Nogue, F.; Robaglia, C.; Gallois, J.L. Mimicking natural polymorphism in eIF4E by CRISPR-Cas9 base editing is associated with resistance to potyviruses. Plant Biotechnol. J. 2019, 17, 1736-1750. [CrossRef] [PubMed] 